\title{
BMJ Open Determinants of community pharmacists' quality of care: a population-based cohort study using pharmacy administrative claims data
}

Nancy Winslade, ${ }^{1,2}$ Robyn Tamblyn ${ }^{3}$

To cite: Winslade N, Tamblyn R. Determinants of community pharmacists' quality of care: a population-based cohort study using pharmacy administrative claims data. BMJ Open 2017;7:e015877. doi:10.1136/ bmjopen-2017-015877

- Prepublication history for this paper is available online. To view these files please visit the journal online (http://dx.doi. org/10.1136/bmjopen-2017015877).

Received 10 January 2017 Revised 2 June 2017 Accepted 19 July 2017

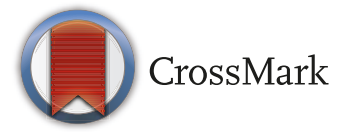

${ }^{1}$ Department of Medicine, McGill University, Montreal, Quebec, Canada

${ }^{2}$ Winslade Consultants, Ottawa, Ontario, Canada

${ }^{3}$ Department of Epidemiology and Biostatistics, McGill University, Montreal, Quebec, Canada

Correspondence to Dr Nancy Winslade; nancy.winslade@mcgill.ca

\section{ABSTRACT}

Objective To determine if a prototype pharmacists services evaluation programme that uses linked community pharmacy claims and health administrative data to measure pharmacists' performance can be used to identify characteristics of pharmacies providing higher quality of care.

Design Population-based cohort study using community pharmacy claims from 1 November 2009 to 30 June 2010 Setting All community pharmacies in Quebec, Canada. Participants 1742 pharmacies dispensing 8655348 antihypertensive prescriptions to 760700 patients. Primary outcome measure Patient adherence to antihypertensive medications.

Predictors Pharmacy level: dispensing workload, volume of pharmacist-provided professional services (eg, refusals to dispense, pharmacotherapy recommendations), pharmacy location, banner/chain, pharmacist overlap and within-pharmacy continuity of care. Patient level: sex, age, income, patient prescription cost, new/chronic therapy, single/multiple antihypertensive medications, single/multiple prescribers and single/multiple dispensing pharmacies. Dispensing level: prescription duration, time of day dispensed and antihypertensive class. Multivariate alternating logistic regression estimated predictors of the primary outcome, accounting for patient and pharmacy clustering.

Results $9.2 \%$ of dispensings of antihypertensive medications were provided to non-adherent patients. Male sex, decreasing age, new treatment, multiple prescribers and multiple dispensing pharmacies were risk factors for increased non-adherence. Pharmacies that provided more professional services were less likely to dispense to nonadherent hypertensive patients (OR: $0.60 ; 95 \% \mathrm{Cl}: 0.57$ to 0.62 ) as were those with better scores on the WithinPharmacy Continuity of Care Index. Neither increased pharmacists' services for improving antihypertensive adherence per se nor increased pharmacist overlap impacted the odds of non-adherence. However, pharmacist overlap was strongly correlated with dispensing workload. There was significant unexplained variability among pharmacies belonging to different banners and chains. Conclusions Pharmacy administrative claims data can be used to calculate pharmacy-level characteristics associated with improved quality of care. This study supports the importance of pharmacist's professional services and continuity of pharmacist's care.

\section{Strengths and limitations of this study}

- The trial directly measured community pharmacy characteristics using pharmacy claims and health administrative data.

- The primary quality of care outcome used a standardised method for measuring patient adherence to medications.

- The trial was population based and included a large sample of patients from community pharmacies in Quebec.

- Performance on only one quality of medication-use indicator was evaluated and results may not apply to additional measures of pharmacists' quality of care.

- Administrative data are limited in the extent to which they can measure services provided by pharmacists that were not billed.

\section{INTRODUCTION}

\section{Background}

Misuse of prescription medications, ranging from inappropriate prescribing to patient non-adherence, remains a significant and costly challenge to health systems. ${ }^{1}$ The medication-related expertise and accessibility of community pharmacists have led policy-makers to re-evaluate the role community pharmacists play in managing medication misuse. ${ }^{23}$ Emphasis has been placed on the care provided by pharmacists both as part of medication dispensing and via expanded professional services that target specific medication-misuse problems. ${ }^{45}$ Although such care can improve patient's medication use, community pharmacists struggle to incorporate expanded professional services into their daily practice. ${ }^{67}$ As a result, payers continue to seek evidence of the real-world impact of community pharmacists' services on medication misuse, ${ }^{489}$ and quality indicators of unsafe or interacting medications and management of non-adherent patients have been established as standardised outcome 
measures of pharmacists' quality of care. ${ }^{10-12}$ The services that pharmacists provide to achieve high performance on these quality indicators can vary across jurisdictions. ${ }^{5}$ Developments in the use of community pharmacy administrative claims data have enabled the measurement of both pharmacy-level performance on these standardised quality indicators and the impact of pharmacists' professional services on patient outcomes. ${ }^{1314}$

To date there have been no precise methods of determining pharmacy-level characteristics that consistently support high levels of pharmacists' performance and that could inform directions for pharmacy policy. Pharmacy characteristics such as workload, continuity of care, culture, workflow and overlap of pharmacists have been evaluated through self-report and with varying definitions of quality performance. ${ }^{15-17}$ The few studies that used standardised quality measures employed a potentially biased ecological approach to estimate pharmacy characteristics by determining a population-based quality metric in the geographical area and then assigning these population-based results to all pharmacies within that area. ${ }^{18-21}$ More robust methodologies are needed to measure the characteristics of the patient, pharmacy and workload situation when the patient receives the medication. ${ }^{22}$

One potentially powerful option is to use pharmacy administrative claims data to measure salient pharmacy characteristics. Until now use of such data has been limited to identifying whether the pharmacy is a chain or independent, and the volume of dispensing. ${ }^{20}{ }^{23}$ This is primarily due to challenges in using the large volume of pharmacy administrative data to create accurate measures, as well as challenges linking pharmacy claims data to other health administrative databases to obtain information on patient and pharmacy characteristics. Increasingly these linkages have been enabled through interest by payers in monitoring performance and researchers in conducting population-based studies. ${ }^{24}{ }^{25}$ We developed a framework for pharmacists' services evaluation that uses linked pharmacy administrative claims and health administrative data to measure and feed back pharmacy-level performance on quality indicators, followed by diagnostic on-site assessments of lower performing pharmacies. ${ }^{26}$ The objective of this study was to determine if the linked administrative health data used within this prototype pharmacists' services evaluation programme could be used to identify characteristics of pharmacies providing higher quality of care.

\section{METHODS \\ Setting}

This study was conducted in Quebec, with a population of 8 million patients of whom approximately 3.5 million receive government support for payment of their medications via the Régie de l'Assurance Maladie du Québec (RAMQ). Since the late 1970s, Quebec pharmacists have been authorised to bill RAMQ for professional services such as refusals to dispense medications and written pharmaceutical opinions for the management of specific medication-use problems. ${ }^{27}{ }^{28} \mathrm{RAMQ}$ requires the date, hour, drug identification number, therapeutic drug class, dosage form, strength, quantity, duration of treatment, specific type and reason for the pharmacist service (eg, previous adverse effect or management of underuse of antihypertensive medications) and costs to RAMQ the patient and for the overall prescription. All data are coded and can be linked to other health administrative data using unique encrypted identifiers for patients, prescribers, pharmacists and pharmacies. For patients, age, sex, postal code and average household income are recorded. For pharmacies, the location (eg, shopping centre) and type of pharmacy (independent or not) are maintained, along with the specific chain or banner to which the pharmacy belongs.

\section{Study design}

A population-based prospective cohort of patients was assembled for whom Quebec pharmacists billed for dispensings of antihypertensive medications between 1 November 2009 to 30 June 2010. A dispensing was defined as the preparation and provision of medications to a patient pursuant to a prescription, regardless of quantity of medication dispensed. Each time there was a dispensing for an antihypertensive medication we determined whether the dispensing was to a patient who was adherent or not over the 90 days prior to the dispensing. Characteristics of each dispensing, the patient and the pharmacy were measured and a multilevel model used to identify predictors of dispensing to a non-adherent patient.

\section{Participants}

All 1891 pharmacies in Quebec were included unless they had opted out of participating in a previously reported randomised controlled trial, were open $<61$ days, or had dispensed $>165317$ prescriptions over the 8-month study period, which represented outliers with Z-scores $>2.5 .{ }^{142}$ Pharmacies with shorter open days did not have sufficient data for reliable calculation of characteristics, and very high dispensing volumes were not representative of traditional community pharmacy practice in Quebec. We had sufficient sample size to have $90 \%$ power to detect a difference in antihypertensive adherence of $5 \%$ for most potential predictors.

\section{Primary outcome}

The primary outcome was whether a dispensing of an antihypertensive medication was provided to an adherent or non-adherent patient. Antihypertensive adherence was selected for this initial evaluation as antihypertensive medications are widely used and non-adherence is common. ${ }^{30}$ Our previous research had also documented that almost all community pharmacies in Quebec $(99.7 \%)$ dispense antihypertensive medications, thereby allowing a population-based cohort for the current study. ${ }^{12}$ 
For each antihypertensive dispensing, we created a record of all dispensings of the same antihypertensive medication to the same patient from all pharmacies in Quebec over the previous 180 days. 'Same medication' was defined as the same drug in the same dosage format, regardless of strength. Switches to a new medication in the same therapeutic class were treated as new therapies. Dispensings of antihypertensive medications were excluded if the patient had not been treated with the same medication for at least 90 days or had not had continuous insurance coverage over the previous 180 days. As dispensing pharmacists are responsible for obtaining information on medications supplied from other pharmacies when determining adherence, each eligible dispensing was attributed to the dispensing pharmacy. We calculated the proportion of previous 90 days covered (PDC) for the same medication using the previous dispensing dates and number of days of supply provided at each dispensing and adjusting for early refills. If the PDC over the 90 days prior to the dispensing was less than 72 days $(80 \%)$, then the dispensing was to a non-adherent patient. ${ }^{31}$

\section{Potential predictors}

Dispensing-level characteristics included the type of antihypertensive medication dispensed, the total prescription cost and the cost to the patient as these have been demonstrated to affect patient adherence. ${ }^{32}$ Although in Quebec the standard supply of medications is for 30 days, patients at risk for non-adherence can receive weekly medication supply and patients stabilised on chronic therapies can receive 90-day supplies. Adherence was, therefore, expected to be worse for patients receiving less than 30 days' supply and better for patients receiving more than 30 days' supply.

Patient-level characteristics were those known to affect adherence such as sex, age and income, with older men and patients with higher income anticipated to be more compliant. ${ }^{2033}$ As our previous work indicated that patients within their first 6 months of antihypertensive therapy are less compliant as are those on single drug therapy or receiving their antihypertensive medications from more than one physician or pharmacy, these variables were also included. ${ }^{1234-36}$

Pharmacy-level characteristics included workload as higher numbers of prescriptions dispensed has been identified as a factor limiting community pharmacists' ability to provide professional services ${ }^{37}$ and predisposing to dispensing errors. ${ }^{15} 1738$ Workload has been reported variously as prescriptions dispensed per year, which can readily be determined from administrative claims data, to prescriptions per pharmacist per hour, which has only been reported using self-reported estimates. ${ }^{17}$ We received from RAMQ the total number of billings and open days for each pharmacy over the 8-month study period and used the administrative claims data to calculate for each pharmacy the average number of: open hours per day, pharmacists billing per hour, prescriptions dispensed per hour and prescriptions dispensed per pharmacist per hour. Related to workload, as medication dispensing errors occur more frequently when only one pharmacist is working, there have been calls for mandatory overlapping of pharmacists' schedules to allow one pharmacist to focus uninterruptedly on prescription verification while a second pharmacist provides professional services. ${ }^{16} 39$ To measure pharmacist overlap for each pharmacy, we created a matrix of the number of pharmacists billing each open hour over each open day during the 8-month study period. From this, we calculated the average per cent of each pharmacy's open hours where more than one pharmacist was billing (Pharmacist Overlap Index). Finally, although continuity of care measuring whether patients received all antihypertensive medications from a single pharmacy was included as a patient-level variable, based on evidence from other health professions that care from the same healthcare professional is important in creating trust, professional relationships, we determined the likelihood that a patient would be cared for by the same pharmacist on multiple visits (Within-Pharmacy Continuity of Care Index).$^{34}$ We calculated, for each pharmacy, the total number of pharmacists working over the 8-month study period (weighted to emphasise differences in high and low numbers of pharmacists) and divided this by the average number of pharmacists working per day at that pharmacy. The lowest value of the index is 1 , representing the best within-pharmacy continuity of care when there is only one single pharmacist working in the pharmacy over the 8 months. Increasing indices indicate a lower chance that the patient would be cared for by the same pharmacist at multiple visits. To determine the culture within the pharmacy, we calculated the total number of pharmacists' professional services billed per 100 prescriptions dispensed over the 8-month period, including refusals to dispense, pharmaceutical opinions, transmission of medication profiles and emergency contraception. We also counted the number of professional services billed specifically for management of underuse of antihypertensive medications.

\section{Data sources/measurement}

Baseline community pharmacy claims data for all dispensings of antihypertensive medications and pharmacist services were received from RAMQ for all Quebec community pharmacies for the period of 1 October 2008 to 30 June 2010. ${ }^{14}$ Patient, pharmacy, pharmacy chain/ banner group, pharmacist and prescriber identifiers were anonymised by RAMQ prior to data transfer. Data for the 8-month period of 1 November 2009 to 30 June 2010 were used to calculate dispensing, patient and pharmacy-level characteristics and estimate determinants of non-adherence.

\section{Statistical methods}

Descriptive statistics summarised the characteristics of the dispensings, patients and pharmacies including the incidence of dispensing to non-adherent patients by type of 
antihypertensive, patient sex and age. Multivariate alternating logistic regression (ALR) estimated the association among the dispensing, patient and pharmacy-level characteristics and non-adherence. ALR allows analysis of dichotomous outcomes when observations have more than one level of clustering. ${ }^{40}$ For our results, ALR first measured the extent of clustering of non-adherence among multiple dispensings within the same patient and then for multiple patients receiving their medications from the same pharmacy. All analyses were completed using SAS V.9.4, with ALR using PROC GENMOD.

Where multiple measures could be calculated to reflect a single construct, results for each measure were first compared with previously reported estimates (if available) to test the accuracy of the calculations. Next, each measure was tested individually for association with non-adherence. A single measure of each construct was selected for inclusion based on the accuracy of the calculation, the strength of evidence supporting its use and the strength of association. Collinearity was evaluated for all variables considered for the final analysis using the variance inflation factor. When collinearity was present, variables that were calculated as interim steps were considered for exclusion and the variables retained were those most directly measuring the constructs of interest. To account for interactions between patient income and the cost of the medication to the patient, we divided both variables into low, medium and high categories and created dummy variables for each of the nine possible interactions, setting low income and low cost to the patient as the reference. ${ }^{41}$

\section{RESULTS}

\section{Study participants}

A total of 1872 pharmacies were enrolled in the study, after $19(1 \%)$ opted out of the previous trial (figure 1). Ninety-one pharmacies open for $<61$ days and 39 additional pharmacies dispensing $>165317$ prescriptions over the 8-month period were removed from the analysis. Evaluation was carried out for 8655348 dispensings of antihypertensive medications to 760700 patients in 1742 pharmacies

\section{Population characteristics}

Angiotensin receptor blockers (ARBs) were the most commonly dispensed antihypertensive medications (23.2\% of dispensings) with $<1 \%$ of dispensings for each of alpha agonists, alpha blockers, potassium sparing diuretics and vasodilators (table 1). Most prescriptions were dispensed in the morning and were for an approximate 1-month duration. A single physician prescribed antihypertensive medications to $74.1 \%$ of patients and $86.0 \%$ went to a single pharmacy for all of their antihypertensive medications over the previous 6 months. Most patients had been taking antihypertensive medications for more than 6 months $(98.5 \%)$ and were on multiple antihypertensive medications $(79.4 \%)$. The majority of pharmacies were either chains or banners $(89.9 \%)$. Pharmacists dispensed an average 18.4 prescriptions per pharmacist per hour, billing for 0.18 professional services for every 100 prescriptions dispensed. Most pharmacies did not have any billings for pharmacists' services for antihypertensive non-adherence, leading to an average of less than one billing over the 8 months $(0.35 \pm 1.8)$. Pharmacies

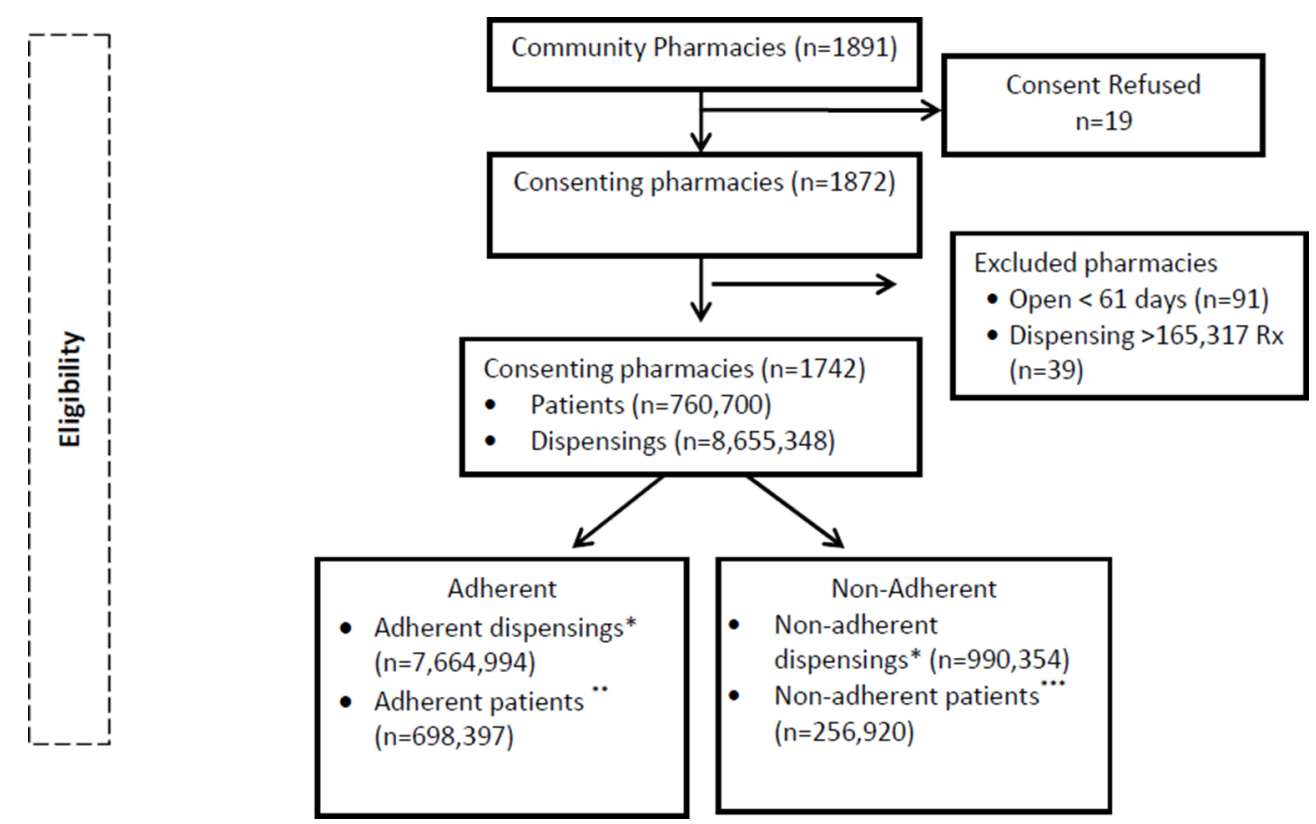

Figure 1 Consort diagram. *Dispensings that were provided to patients who had been either adherent or non-adherent with their antihypertensive medication over the previous 90 days. ${ }^{\star *}$ Patients with at least one adherent dispensing over the 8-month study period. ${ }^{* \star *}$ Patients with at least one non-adherent dispensing over the 8-month study period. As patients received multiple dispensings, they could be counted as both adherent and non-adherent; therefore, the total of adherent and non-adherent patients is more than 760700 . 
Table 1 Characteristics of prescriptions dispensed, patients and their pharmacies

Level of characteristic $\mathbf{n}(\%)$

Dispensed prescription level $(\mathrm{N}=\mathbf{8 6 5 5 3 4 8 )}$

Time of day dispensed

\begin{tabular}{ll} 
Morning (>8 noon) & $4273894(49.4)$ \\
\hline Afternoon (>noon-16) & $3141594(36.3)$ \\
\hline Evening $(>16-20)$ & $1065102(12.3)$ \\
Overnight $(>20-8)$ & $174758(2.0)$ \\
No of days of medication supplied & \\
$<10$ & $180524(2.1)$ \\
$10-32$ & $8241026(95.2)$ \\
$>32$ & $233798(2.7)$
\end{tabular}

Type of antihypertensive medication dispensed

\begin{tabular}{ll} 
Angiotensin receptor blockers & $2004146(23.2)$ \\
Beta-blockers & $1853835(21.4)$ \\
Calcium channel blockers & $1828320(21.1)$ \\
ACE inhibitors & $1391246(16.1)$ \\
\hline Thiazide diuretics & $672041(7.8)$ \\
Loop diuretics & $368466(4.3)$ \\
Diuretic combinations & $184101(2.1)$ \\
Other diuretics & $145051(1.7)$ \\
Alpha agonists & $74278(0.9)$ \\
Alpha blockers & $68367(0.8)$ \\
Potassium sparing diuretics & $56693(0.7)$ \\
Vasodilators & $8804(0.1)$ \\
Cost & Mean (SD) \\
\hline Total cost of the prescription $(\$ C)$ & $\$ C 28.36(\$ C 17.48)$ \\
Cost to the patient of the & $\$ C 8.55(\$ C 8.56)$ \\
prescription $(\$ C)$ &
\end{tabular}

Pharmacy client level* $(\mathrm{N}=760700)$

Sex

\begin{tabular}{ll} 
Female & $4858885(56.1)$ \\
Male & $3800463(43.9)$ \\
Age (years) & \\
$<65$ & $2055518(23.8)$ \\
$65-69$ & $1595657(18.4)$ \\
$70-79$ & $3106633(35.9)$ \\
$>79$ & $1897540(21.9)$ \\
Income & \\
Low (<\$C31 700) & $647805(7.5)$ \\
Middle (\$C31 700-80 000) & $7096041(82.0)$ \\
High (>\$C80 000) & $911502(11.5)$ \\
Antihypertensive therapy & $126812(1.5)$ \\
New therapy (<6 months) & $8528536(98.5)$ \\
Chronic therapy ( $\geq 6$ months) & $1782490(20.6)$ \\
\hline Single antihypertensive drug &
\end{tabular}

Continued
Table 1 Continued

\begin{tabular}{|c|c|}
\hline Level of characteristic & n (\%) \\
\hline Multiple antihypertensive drugs & $6872858(79.4)$ \\
\hline \multicolumn{2}{|l|}{ Continuity of care } \\
\hline $\begin{array}{l}\text { Single pharmacy dispensed } \\
\text { antihypertensives over previous } \\
6 \text { months }\end{array}$ & $7440825(86.0)$ \\
\hline $\begin{array}{l}\text { Multiple pharmacies dispensed } \\
\text { antihypertensives over previous } \\
6 \text { months }\end{array}$ & 1214523 (14.0) \\
\hline $\begin{array}{l}\text { Single prescriber of } \\
\text { antihypertensives over previous } \\
6 \text { months }\end{array}$ & $6412928(74.1)$ \\
\hline $\begin{array}{l}\text { Multiple prescribers of } \\
\text { antihypertensives over previous } \\
6 \text { months }\end{array}$ & $2242420(25.9)$ \\
\hline
\end{tabular}

\section{Community pharmacy level ( $\mathrm{N}=1742)$}

\begin{tabular}{|c|c|}
\hline Pharmacy type & $\mathrm{n}(\%)$ \\
\hline Chain/banner & $1566(89.9)$ \\
\hline Independent & $176(10.1)$ \\
\hline \multicolumn{2}{|l|}{ Pharmacy location } \\
\hline Neighbourhood pharmacy & $457(26.2)$ \\
\hline Shopping centre & $281(16.1)$ \\
\hline Medical clinic & $283(16.2)$ \\
\hline Other & $53(3.1)$ \\
\hline Missing & $668(38.3)$ \\
\hline
\end{tabular}

Professional services provided over 8 months

Total pharmacist services billed per 100 prescriptions

$\begin{array}{ll}<0.12 & 544(31.2) \\ 0.13-0.2 & 588(33.8) \\ >0.2 & 610(35.0)\end{array}$

Recommendations for non-adherence with antihypertensive medications

$\begin{array}{cl}0 & 1485(85.3) \\ 1-5 & 237(13.6) \\ 6-10 & 17(0.1) \\ >10 & 3(0.2)\end{array}$

Workload Mean (SD)

Total prescriptions dispensed over 53308 (36749) 8 months

\begin{tabular}{ll} 
Total days open over 8months & $214(42.8)$ \\
\hline Hours open per day & $14.4(3.3)$ \\
\hline Pharmacists working/day & $1.8(0.7)$ \\
Pharmacists working/hour & $1.1(0.1)$ \\
Prescriptions dispensed/day & $244.6(156.6)$ \\
Prescriptions dispensed/hour & $20.5(13.0)$ \\
Prescriptions dispensed/ & $18.4(10.5)$ \\
pharmacist/hour &
\end{tabular}

Continued 


\begin{tabular}{|c|c|}
\hline Level of characteristic & n (\%) \\
\hline $\begin{array}{l}\text { Pharmacist Overlap Index } \\
\text { (average per cent of open hours } \\
\text { with }>1 \text { pharmacist) }\end{array}$ & $15.48(9.14)$ \\
\hline \multicolumn{2}{|l|}{ Within-pharmacy continuity of care (COC) } \\
\hline $\begin{array}{l}\text { Distinct pharmacists employed over } \\
8 \text { months }\end{array}$ & $9.0(6.7)$ \\
\hline $\begin{array}{l}\text { Within-Pharmacy COC Index } \\
\text { (weighted no of pharmacist in } \\
8 \text { months/no of pharmacists per day) }\end{array}$ & $17.3(20.1)$ \\
\hline
\end{tabular}

${ }^{*}$ Considering all patients who received eligible dispensings over 8 months' follow-up.

had more than one pharmacist billing for $15.5 \%$ of their open hours and an average of nine different pharmacists worked in each pharmacy over the 8-month study period.

\section{Non-adherence}

Over 8 months, $9.2 \%$ of all dispensings of antihypertensive medications were provided to non-adherent patients (795031 of 8655348 dispensings) (table 2). Antihypertensive dispensings were provided to 760700 distinct patients, $31 \%$ of whom were non-adherent to their antihypertensive medication at least once over the study period (235885 of $760700)$. The highest incidence of non-adherence occurred with alpha agonists $(21.49 \%)$ and for dispensings provided in the evening (12.03\%). The incidence of non-adherence was also higher if the patient was $<65$ years old $(12.41 \%)$, new to therapy $(18.29 \%)$ or on a single antihypertensive medication $(12.47 \%)$.

When adjusted for the three levels of variables and clustering, the odds of non-adherence were significantly greater for medications supplied for less than 10 days and for medications dispensed at times other than morning $(p<0.05)$ (table 2). Relative to beta-blockers, the odds of dispensing an ARB or ACE inhibitor to a non-adherent patient were decreased by $17 \%$ (OR: $0.83 ; 95 \%$ CI: 0.82 to 0.84 ).

Older, female patients were less likely to be non-adherent at the time of receiving an antihypertensive medication, with a $41 \%$ decrease in the odds for patients $\geq 80$ years relative to patients $<65$ years old (OR: 0.59 ; $95 \%$ CI: 0.58 to 0.60 ). Patients newly started on their antihypertensive medication within the past 6 months experienced a $27 \%$ increase in odds of non-adherence at the time of dispensing. Patients with decreased continuity of care were also more likely to be non-adherent at the time of dispensing, with the odds of non-adherence increased by $10 \%$ if the patient had used multiple pharmacies and $16 \%$ if she/he had used multiple physicians for their antihypertensive medications over the past 6 months. The impact of cost of the medication to the patient was modified by the patient's income and, in contrast to the unadjusted incidence of non-adherence where increasing out-of-pocket costs lead to higher non-adherence, when adjusted for all three levels of characteristics, higher out-of-pocket costs resulted in a decreased odds of non-adherence within all of low-income, middle-income and high-income patients. High-income patients with low out-of-pocket medication costs were $15 \%$ more likely to be non-adherent at the time of dispensing as compared with low-income patients with low medication costs (OR: 1.15, 95\% CI: 1.12 to 1.18 ).

At the pharmacy level, the odds of non-adherence decreased by $40 \%$ per 1 increase in the number of professional services billed per 100 prescriptions dispensed (OR: $0.60 ; 95 \%$ CI: 0.57 to 0.62 ). Neither the number of billings for pharmacists' services targeted at managing non-adherence with antihypertensive medications nor the percentage of open hours with overlapping pharmacists influenced non-adherence. However, pharmacist overlap was highly correlated with dispensing volume (Pearson correlation coefficient 0.51, $\mathrm{p}<0.0001$ ). Higher workload decreased the odds of non-adherence by $4 \%$ per 10 prescription increase in number of prescriptions dispensed per pharmacist per hour (OR: 0.96; 95\% CI: 0.96 to 0.97$)$. Higher scores on the Within-Pharmacy Continuity Care Index, indicating a decreased chance of patients being cared for by the same pharmacist, slightly but significantly increased the odds of non-adherence (OR: $1.003 ; 95 \%$ CI: 1.001 to 1.005 ). There was significant variability in the odds of non-adherence among pharmacies belonging to various banners or chains and the odds of non-adherence were significantly higher for chains/ banners relative to independent pharmacies (OR: 1.02; 95\% CI: 1.00 to 1.05 ).

\section{DISCUSSION}

\section{Statement of principal findings}

This study is the first to document that linked community pharmacy claims and health administrative data can be used to directly measure a range of pharmacy-level characteristics and quality measures. It is also the first study that investigated the association between the provision of pharmacists' professional services and better within-pharmacy continuity of care with adherence, showing that each of these pharmacists' practices are associated with a decreased odds of dispensing antihypertensive medications to non-adherent patients.

\section{Strengths and limitations}

The main strengths of this study are the direct measurement of pharmacy characteristics from administrative claims data and the use of an objective, validated quality of care measure of adherence. ${ }^{10-12}$ As significant variability in results has been reported from studies using differing measures of adherence, use of standardised methods for measuring adherence is particularly important in determining predictors of non-adherence. ${ }^{10}$ As only $1 \%$ of community pharmacies in Quebec did not consent to participate (18 of 1891), a second strength is that the sample approximated a population-based cohort and selection bias was minimised. Limitations include that we evaluated performance on only one 
Table 2 Dispensed prescription, patient and pharmacy characteristics associated with risk of non-adherence with antihypertensive medications

\begin{tabular}{|c|c|c|c|c|c|}
\hline & $\mathbf{n}$ & Non-adherence & Multivariat & rnating logis & regression \\
\hline & & (\%) & OR & $95 \% \mathrm{Cl}$ & p Value \\
\hline \multicolumn{6}{|l|}{ Dispensed prescription level } \\
\hline All dispensings & 8655348 & 9.19 & & & \\
\hline \multicolumn{6}{|l|}{ Time of day dispensed } \\
\hline Morning (8-noon) & 4273894 & 7.89 & Reference & & \\
\hline Afternoon (noon-16) & 3141594 & 9.86 & 1.03 & 1.03 to 1.04 & $<0.0001$ \\
\hline Evening (16-20) & 1065102 & 12.03 & 1.06 & 1.05 to 1.06 & $<0.0001$ \\
\hline Overnight (20-8) & 174758 & 11.37 & 1.03 & 1.02 to 1.05 & $<0.0001$ \\
\hline \multicolumn{6}{|l|}{ No of days supplied } \\
\hline 10-32 & 8241026 & 9.10 & Reference & & \\
\hline$<10$ & 180524 & 8.12 & 1.16 & 1.12 to 1.19 & $<0.0001$ \\
\hline$>32$ & 233798 & 13.13 & 0.84 & 0.82 to 0.86 & $<0.0001$ \\
\hline \multicolumn{6}{|l|}{ Type of antihypertensive } \\
\hline Beta-blockers & 1853835 & 9.16 & Reference & & \\
\hline Angiotensin receptor blockers & 2004146 & 8.63 & 0.83 & 0.82 to 0.84 & $<0.0001$ \\
\hline Calcium channel blockers & 1828320 & 8.93 & 0.98 & 0.97 to 0.99 & $<0.0001$ \\
\hline ACE inhibitors & 1391246 & 8.13 & 0.83 & 0.83 to 0.84 & $<0.0001$ \\
\hline Thiazide diuretics & 672041 & 9.51 & 0.98 & 0.97 to 0.99 & $<0.0001$ \\
\hline Loop diuretics & 368466 & 12.70 & 1.50 & 1.48 to 1.52 & $<0.0001$ \\
\hline Diuretic combinations & 184191 & 12.23 & 1.19 & 1.17 to 1.22 & $<0.0001$ \\
\hline Other diuretics & 145051 & 8.28 & 0.89 & 0.87 to 0.91 & $<0.0001$ \\
\hline Alpha agonists & 74278 & 21.49 & 2.71 & 2.63 to 2.79 & $<0.0001$ \\
\hline Alpha blockers & 68367 & 8.72 & 1.12 & 1.08 to 1.15 & $<0.0001$ \\
\hline Potassium sparing diuretics & 56693 & 13.44 & 1.28 & 1.24 to 1.32 & $<0.0001$ \\
\hline Vasodilators & 8804 & 15.19 & 1.87 & 1.70 to 2.05 & $<0.0001$ \\
\hline \multicolumn{6}{|l|}{ Patient characteristics } \\
\hline \multicolumn{6}{|l|}{ Sex } \\
\hline Male & 3800463 & 9.69 & Reference & & \\
\hline Female & 4854885 & 8.79 & 0.90 & 0.90 to 0.92 & $<0.0001$ \\
\hline \multicolumn{6}{|l|}{ Age (years) } \\
\hline$<65$ & 2055518 & 12.41 & Reference & & \\
\hline $65-69$ & 1595657 & 8.70 & 0.66 & 0.64 to 0.66 & $<0.0001$ \\
\hline $70-79$ & 3106633 & 8.02 & 0.60 & 0.59 to 0.61 & $<0.0001$ \\
\hline$\geq 80$ & 1897540 & 8.00 & 0.59 & 0.48 to 0.60 & $<0.0001$ \\
\hline \multicolumn{6}{|l|}{ Patient income ${ }^{*}$ patient cost interaction } \\
\hline Low income and low cost & 301826 & 8.67 & Reference & & \\
\hline Low income and middle cost & 184565 & 9.59 & 0.93 & 0.91 to 0.95 & $<0.0001$ \\
\hline Low income and high cost & 161414 & 9.89 & 0.88 & 0.87 to 0.90 & $<0.0001$ \\
\hline Middle income and low cost & 2286651 & 8.47 & 0.99 & 0.97 to 1.01 & 0.241 \\
\hline Middle income and middle cost & 2459139 & 9.28 & 0.97 & 0.95 to 0.99 & 0.003 \\
\hline Middle income and high cost & 2350251 & 9.27 & 0.95 & 0.93 to 0.97 & $<0.0001$ \\
\hline High income and low cost & 210972 & 10.31 & 1.15 & 1.12 to 1.18 & $<0.0001$ \\
\hline High income and middle cost & 339456 & 10.53 & 1.07 & 1.04 to 1.09 & $<0.0001$ \\
\hline High income and high cost & 361074 & 10.50 & 1.01 & 0.99 to 1.04 & 0.336 \\
\hline
\end{tabular}


Table 2 Continued

\begin{tabular}{cllcc}
$\mathrm{n}$ & Non-adherence & Multivariate alternating logistic regression \\
\hline & $(\%)$ & OR & $95 \% \mathrm{Cl}$ & $\mathrm{p}$ Value
\end{tabular}

Antihypertensive therapy

\begin{tabular}{|c|c|c|c|c|c|}
\hline Chronic therapy ( $\geq 6$ months) & 8528536 & 9.05 & Reference & & \\
\hline New therapy (<6 months) & 126812 & 18.29 & 1.27 & 1.25 to 1.30 & $<0.0001$ \\
\hline Multiple antihypertensive drugs & 6872858 & 8.33 & Reference & & \\
\hline Single antihypertensive drug & 1782490 & 12.47 & 1.04 & 1.04 to 1.05 & $<0.0001$ \\
\hline \multicolumn{6}{|l|}{ Continuity of care } \\
\hline Single dispensing pharmacy & 7440825 & 8.86 & Reference & & \\
\hline Multiple dispensing pharmacies & 1214523 & 11.16 & 1.10 & 1.08 to 1.11 & $<0.0001$ \\
\hline Single prescriber & 6412928 & 8.65 & Reference & & \\
\hline Multiple prescribers & 2242420 & 10.72 & 1.16 & 1.15 to 1.17 & $<0.0001$ \\
\hline
\end{tabular}

Pharmacy characteristics

Pharmacy type

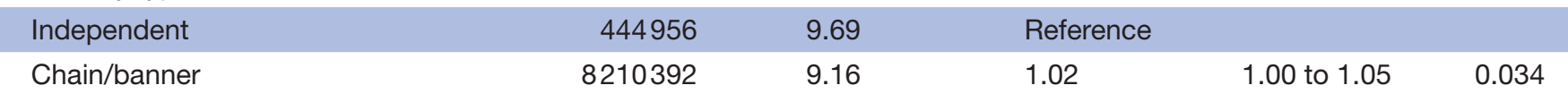

Anonymised pharmacy chain/banner/independent

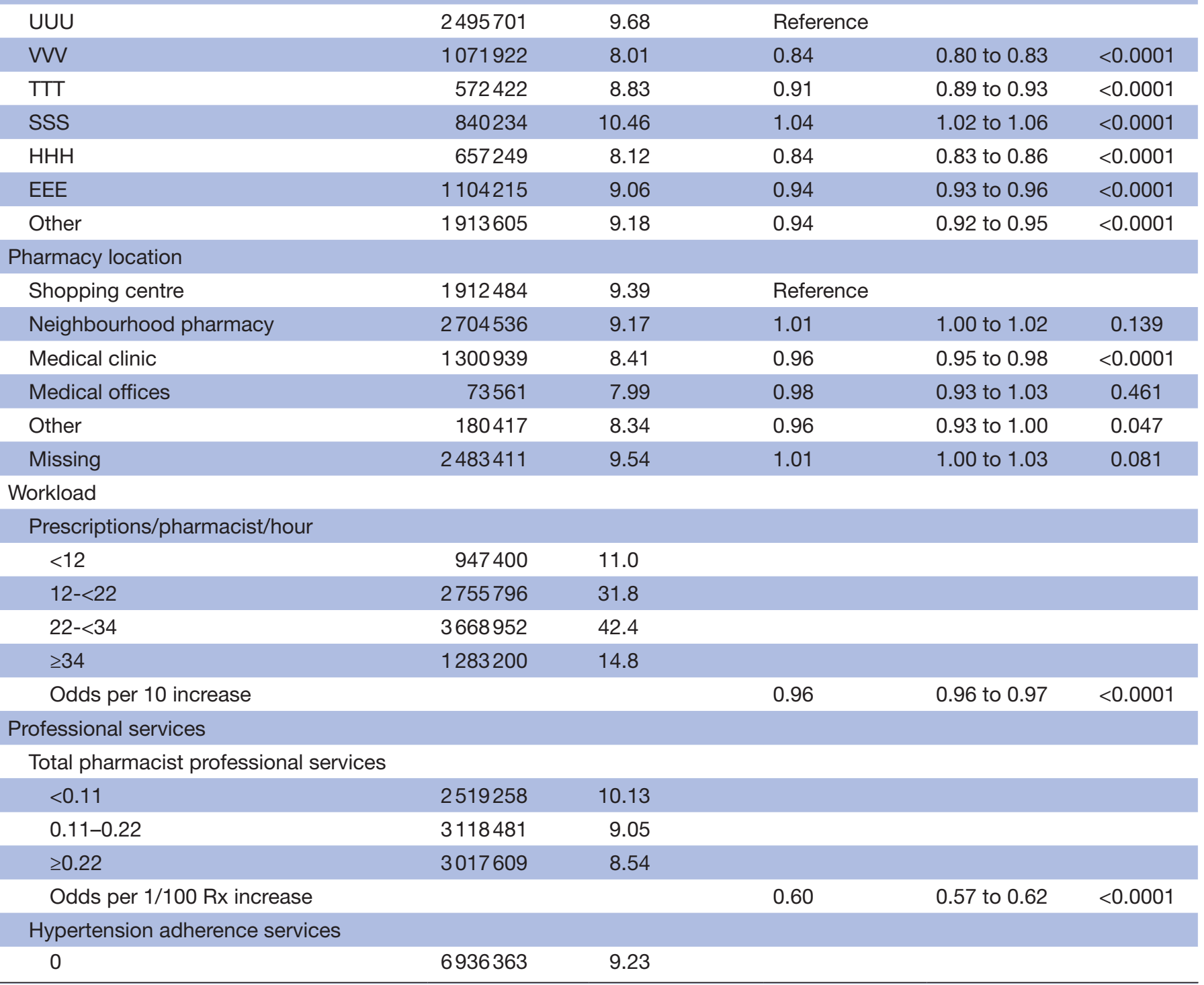


Table 2 Continued

\begin{tabular}{|c|c|c|c|c|c|}
\hline & \multirow[t]{2}{*}{$\mathbf{n}$} & \multirow{2}{*}{$\begin{array}{l}\text { Non-adherence } \\
(\%)\end{array}$} & \multicolumn{3}{|c|}{ Multivariate alternating logistic regression } \\
\hline & & & OR & $95 \% \mathrm{Cl}$ & p Value \\
\hline $1-5$ & 1553820 & 8.95 & & & \\
\hline$\geq 10$ & 19772 & 8.74 & & & \\
\hline Odds per 1 per 8 month increase & & & 1.00 & 1.00 to 1.00 & 0.083 \\
\hline \multicolumn{6}{|l|}{ Pharmacist overlap index } \\
\hline$<10 \%$ & 1242727 & 14.4 & & & \\
\hline $10 \% \leq 16 \%$ & 2780707 & 32.1 & & & \\
\hline $16 \% \leq 22 \%$ & 1532245 & 17.7 & & & \\
\hline$\geq 22 \%$ & 3099669 & 35.8 & & & \\
\hline$>5-10$ & 2554425 & 8.93 & & & \\
\hline$>10-20$ & 2331227 & 9.37 & & & \\
\hline$>20$ & 2486765 & 9.50 & & & \\
\hline Odds per 10 increase & & & 1.003 & 1.001 to 1.005 & 0.012 \\
\hline
\end{tabular}

quality of medication-use measure and results cannot be generalised to other measures of pharmacists' quality of care. Although underuse measures of other therapeutic categories such as lipid-lowering or diabetes may show similar results, determinants of performance on quality indicators measuring medication overuse (eg, rescue inhalers for asthma) or unsafe dispensing may differ as the professional services pharmacists provide to detect and manage these medication-use problems differ from those provided for medication underuse. Evaluation of performance on additional quality indicators measuring both adherence and unsafe dispensing is required to determine if results are generalizable. In addition, our methodology for calculating adherence did not allow for detection of primary non-adherence or non-adherence/ non-persistence within the first 90 days of therapy. As these types of non-adherence are problematic with antihypertensive medications, our results may have underestimated non-adherence and measures of these additional types of non-adherence should be evaluated. Finally, administrative claims data are limited in the extent to which they can measure whether pharmacists provided a service but did not bill for it. ${ }^{42-44}$

\section{Interpretation}

Our overall rate of non-adherence is consistent with previous reports that use community pharmacy administrative claims data and similar measures of non-adherence. ${ }^{1045}$ Calculation of pharmacy-level characteristics required multiple steps and complex analysis and for characteristics that had previously been estimated via self-report, such as prescriptions per pharmacist per hour, our results were higher $(18.4 \pm 10.5$ our study vs
$14.1 \pm 4.9){ }^{17}$ This is consistent with national reports documenting higher total prescriptions dispensed in Quebec relative to other provinces. ${ }^{37}$ Results of the drug and patient characteristics affecting non-adherence agree with previous research documenting that there is higher adherence to antihypertensive medications with fewer side effects, such as ARB and ACE, and that increasing age is associated with increased adherence to antihypertensive medications. ${ }^{3246}$ However, given the variability in results of non-adherence rates and predictors from studies that used differing measurement methodologies, our results should be compared with studies using pharmacy administrative claims data and standardised methods for measuring non-adherence. ${ }^{10}$ To our knowledge, this literature is limited to the study that used an ecological approach to measuring pharmacy and patient characteristics. ${ }^{20}$ Our results differ from this ecological study for the impact of patient sex and income, and independent pharmacy ownership on the odds of dispensing to a non-adherent patient. Our results demonstrate the impact of measuring these characteristics directly for each dispensing and adjusting for clustering. When only considering whether the pharmacy is independent versus a chain/ banner, the incidence of non-adherence is higher in independent pharmacies. However, when adjusted for clustering and the remaining dispensing, patient and pharmacy characteristics, this association reverses with chain/banner pharmacies demonstrating a greater odds of non-adherence. The same is true for the impact of patient costs relative to income. Without adjustment, the incidence of non-adherence increases as cost to the 
patient increases. However, when adjusted for all characteristics, this relationship reverses. As higher patient cost typically occurs with second-line treatments for hypertension, this may represent patients who required switches or additions to their therapies due to side or insufficient effects from their initial treatments, which has been shown to increase adherence. ${ }^{47}$

The most striking results of our analysis are the reductions in the odds of non-adherence with both an increasing rate of provision of pharmacists' professional services and improved within-pharmacy continuity of care. It is hypothesised that the relationship between the rate of provision of these services and lower non-adherence indicates that improved quality of care is provided at pharmacies where pharmacists prioritise provision professional services versus involvement in technical distributive functions. ${ }^{48} 49$ The relationship between improved within-pharmacy continuity of care and decreased odds of non-adherence supports such a hypothesis as patients can more easily develop trusting relationships with their pharmacist when continuity of care is improved. Our findings that increased workload is associated with lower odds of non-adherence would not appear to support that increased workload challenges pharmacists' provision of quality care. However, we had removed very high-volume pharmacies so we did not see the previously reported results of lower quality of care in pharmacies with both very low and very high dispensing volumes. ${ }^{15}$ The strong positive correlation between workload and pharmacist overlap suggests that pharmacists are not being scheduled to provide professional services but to enable increased number of prescriptions to be processed. As both culture and workflow are determined predominantly by the pharmacist owner, greater freedom to emphasise professional pharmacists' practice by owners of independent pharmacies could account for their lower odds of non-adherence relative to chains/banners. ${ }^{50}$ Similarly, differences in practical philosophy among the chains/ banners could account for the variability in performance among the different banners and chains.

\section{Implications and future research}

Our results indicate that emphasis on the caring role of pharmacists both during dispensing and via provision of professional services appears key to improving patients' use of medications. Results also support policies that encourage continuity of care and that focus adherence strategies on younger men, new to treatment and taking single antihypertensive therapy. Pharmacy administrative claims data can be used to directly measure dispensing, patient and pharmacy characteristics, thereby increasing the range and accuracy of pharmacy-level characteristics evaluated. Evaluation of additional measures both of non-adherence and dispensing of contraindicated medications is needed to determine if there is consistency across the measures of pharmacy-level characteristics identified in our study as being related to pharmacists' quality of care.
Acknowledgements We thank Danielle Fagnan, from the Quebec Order of Pharmacists, for the support provided throughout the project. We thank Sherry Shi for her analysis of the data. The critical review of the manuscript and recommendations for analysis by Drs Cees van der Vleuten and Lambert Schuwirth are gratefully acknowledged. We acknowledge the financial support of Green Shield Canada Foundation and the financial and administrative support of the Quebec Order of Pharmacists.

Contributors NW and RT conceived and designed the project. NW reviewed literature to determine relevant potential predictors. RT provided expertise on the statistical analysis. NW completed the basic analysis and drafted the manuscript. RT contributed substantially to the manuscript revision. NW redrafted and finalised the manuscript following reviewers' recommendations. RT contributed substantially to the final revisions.

Funding Green Shield Foundation Canada provided a grant for the completion of this project and was both informed of the decision to submit this paper and received a copy of the manuscript. The Quebec Order of Pharmacists provided a matching grant in the form of salary support, facilitated and paid the required data access fees to RAMQ.

Competing interests NW reports grants from Green Shield Foundation Canada, personal fees and non-financial support from Quebec Order of Pharmacists during the conduct of the study. RT reports grants from Green Shield Foundation Canada and non-financial support from Quebec Order of Pharmacists during the conduct of the study.

Ethics approval McGill University's Faculty of Medicine Institutional Review Board.

Provenance and peer review Not commissioned; externally peer reviewed.

Data sharing statement № additional unpublished data are available from this study.

Open Access This is an Open Access article distributed in accordance with the Creative Commons Attribution Non Commercial (CC BY-NC 4.0) license, which permits others to distribute, remix, adapt, build upon this work non-commercially, and license their derivative works on different terms, provided the original work is properly cited and the use is non-commercial. See: http://creativecommons.org/ licenses/by-nc/4.0/

C) Article author(s) (or their employer(s) unless otherwise stated in the text of the article) 2017. All rights reserved. No commercial use is permitted unless otherwise expressly granted.

\section{REFERENCES}

1. Thomas F, Depledge M. Medicine 'misuse': Implications for health and environmental sustainability. Soc Sci Med 2015;143:81-7.

2. Mossialos E, Courtin E, Naci H, et al. From "retailers" to health care providers: Transforming the role of community pharmacists in chronic disease management. Health Policy 2015;119:628-39.

3. Tannenbaum C, Tsuyuki RT. The expanding scope of pharmacists' practice: implications for physicians. CMAJ 2013;185:1228-32.

4. Williams M, Peterson GM, Tenni PC, et al. Drug-related problems detected in Australian Community Pharmacies: The PROMISe Trial. Ann Pharmacother 2011;45:1067-76.

5. Conference Board of Canada for the Canadian Pharmacists Association. A review of pharmacy services in Canada and the health and economic evidence. Ottawa, Ontario, 2016. https:// www.pharmacists.ca/cpha-ca/assets/File/cpha-on-the-issues/ Pharmacy\%20Services\%20Report\%201.pdf (accessed 31 Oct 2016).

6. Pringle JL, Boyer A, Conklin MH, et al. The Pennsylvania Project: pharmacist intervention improved medication adherence and reduced health care costs. Health Aff 2014;33:1444-52.

7. Avery AJ, Rodgers S, Cantrill JA, et al. A pharmacist-led information technology intervention for medication errors (PINCER): a multicentre, cluster randomised, controlled trial and costeffectiveness analysis. Lancet 2012;379:1310-9.

8. National Quality Forum. Preventing Medication Errors: a $\$ 21$ Billion Opportunity. http://www.qualityforum.org/Publications/2010/12/ Preventing_Medication_Errors_CAB.aspx (accessed 31 Oct 2016).

9. Al Hamid A, Ghaleb M, Aljadhey H, et al. A systematic review of hospitalization resulting from medicine-related problems in adult patients. Br J Clin Pharmacol 2014;78:202-17.

10. Dunbar-Jacob J, Rohay JM. Predictors of medication adherence: fact or artifact. J Behav Med 2016;39:957-68. 
11. Pillittere-Dugan D, Nau DP, McDonough $\mathrm{K}$, et al. Development and testing of performance measures for pharmacy services. J Am Pharm Assoc 2009;49:212-9.

12. Winslade N, Taylor L, Shi S, et al. Monitoring community pharmacist's quality of care: a feasibility study of using pharmacy claims data to assess performance. BMC Health Serv Res 2011;11:12.

13. Seabury SA, Lakdawalla DN, Dougherty JS, et al. Medication adherence and measures of health plan quality. Am J Manag Care 2015;21:e379-89.

14. Winslade N, Eguale T, Tamblyn R. Optimising the changing role of the community pharmacist: a randomised trial of the impact of audit and feedback. BMJ Open 2016;6:e010865.

15. Schafheutle El, Seston EM, Hassell K. Factors influencing pharmacist performance: a review of the peer-reviewed literature. Health Policy 2011;102:178-92.

16. Pervanas HC, Revell N, Alotaibi AF. Evaluation of Medication Errors in Community Pharmacy Settings: A Retrospective Report. J of Pharmacy Technology 2016;32:71-4.

17. Malone DC, Abarca J, Skrepnek GH, et al. Pharmacist workload and pharmacy characteristics associated with the dispensing of potentially clinically important drug-drug interactions. Med Care 2007:45:456-62.

18. Morgenstern $\mathrm{H}$. Ecologic studies in epidemiology: concepts, principles, and methods. Annu Rev Public Health 1995;16:61-81.

19. Jackson TH, Bentley JP, McCaffrey DJ, et al. Store and Prescription Characteristics Associated with Primary Medication Non-adherence. J Manag Care Pharm 2014;20:824-32.

20. Desai V, Nau D, Conklin M, et al. Impact of Environmental Factors on Differences in Quality of Medication Use: An Insight for the Medicare Star Rating System. J Manag Care Spec Pharm 2016;22:779-86.

21. Sedgwick P. Understanding the ecological fallacy. BMJ 2015;351:h4773.

22. Dharmarajan S, Bentley JP, Banahan BF III, et al. Measuring Pharmacy Performance in the Area of Medication Adherence: Addressing the Issue of risk assessment. J Manag Care Pharm 2014;20:1057-68.

23. Pechlivanoglou $P$, Abrahamyan $L$, Mackeigan $L$, et al. Factors affecting the delivery of community pharmacist-led medication reviews: evidence from the MedsCheck annual service in Ontario. BMC Health Serv Res 2016;16:666.

24. Centers for Medicare \& Medicaid Services. CMS creates new chief data officer post. https://www.cms.gov/Newsroom/ MediaReleaseDatabase/Press-releases/2014-Press-releases-items/ 2014-11-19.html (accessed 2 Jan 2017).

25. Institute for Clinical Evaluative Sciences (ICES). ICES launces Data and Analytic Services (ICES DAS). http://www.ices.on.ca/Newsroom/ News-Releases/2014/ICES-launches-Data-and-Analytic-ServicesICES-DAS (accessed 2 Jan 2017).

26. Winslade NE, Tamblyn RM, Taylor LK, et al. Integrating performance assessment, maintenance of competence, and continuing professional development of community pharmacists. Am J Pharm Educ 2007;71:15.

27. Kröger E, Moisan J, Grégoire JP. Billing for cognitive services: understanding Québec pharmacists' behavior. Ann Pharmacother 2000;34:309-16.

28. RAMQ. Section 2.3.3.2 Ordonnance et service professionnel. Manuel des pharmaciens http://www.ramq.gouv.qc.ca/fr/professionnels/ pharmaciens/manuels/Pages/manuel-pharmaciens.aspx (accessed 11 May 2017).

29. Cousineau D, Chartier S. Outliers detection and treatment: a review. Int J Psychol Res 2010;3:58-67.

30. Tong X, Chu EK, Fang J, et al. Nonadherence to Antihypertensive Medication Among Hypertensive Adults in the United States-HealthStyles, 2010. J Clin Hypertens 2016;18:892-900.
31. Center for Disease Control and Prevention, National Department of Health \& Human Services. Calculating proportion of days covered (pdc) for antihypertensive and antidiabetic medications: An evaluation guide for grantees. Atlanta, Georgia. https://www.cdc.gov/dhdsp/ docs/med-adherence-evaluation-tool.pdf (accessed 9 May 2017).

32. Tedla YG, Bautista LE. Drug side effect symptoms and adherence to antihypertensive medication. Am J Hyperten 2016;29:772-9.

33. Kardas P, Lewek P, Matyjaszczyk M. Determinants of patient adherence: a review of systematic reviews. Front Pharmacol 2013;4.

34. Christie R. Does continuity of community pharmacy care influence adherence to statins? Halifax, Nova Scotia, 2013. https://dalspace. library.dal.ca/bitstream/handle/10222/42712/Christie-Russell-MScCHESeptember-2013.pdf?sequence=7 (accessed 31 Oct 2016).

35. van Walraven $\mathrm{C}$, Oake $\mathrm{N}$, Jennings $\mathrm{A}$, et al. The association between continuity of care and outcomes: a systematic and critical review. J Eval Clin Pract 2010;16:947-56.

36. DuGoff EH, Bandeen-Roche K, Anderson GF. Relationship between continuity of care and adverse outcomes varies by number of chronic conditions among older adults with diabetes. $J$ Comorbidity 2016;6:65-72.

37. Neighbourhood Pharmacy Association of Canada. Pharmacy 360: the retail pharmacy business in Canada. Toronto, Ontario 2016.

38. National Association of Boards of Pharmacy. Performance Metrics and Quotas in the Practice of Pharmacy, 2013. https://nabp. pharmacy/performance-metrics-and-quotas-in-the-practice-ofpharmacy-resolution-109-7-13/ (accessed 24 Oct 2016).

39. Bradway D. Connecticut Pharmacists Association. Evaluation of pharmacy metric and workload factors impact on patient safety. http://docplayer.net/20086385-Evaluation-of-pharmacy-metric-andworkload-factors-impact-on-patient-safety.html (accessed 31 Oct 2016).

40. Tamblyn R, Eguale T, Huang A, et al. The Incidence and Determinants of Primary Nonadherence with Prescribed Medication in Primary Care. Ann Intern Med 2014;160:441-50.

41. Tamblyn R, Laprise R, Hanley JA, et al. Adverse events associated with prescription drug cost-sharing among poor and elderly persons. JAMA 2001;285:421-9.

42. Arnet I, Abraham I, Messerli M, et al. A method for calculating adherence to polypharmacy from dispensing data records. Int $J$ Clin Pharm 2014;36:192-201.

43. Katz A, Soodeen R-A, Bogdanovic B, et al. Can the quality of care in family practice be measured using administrative data? Health Serv Res 2006;41:2238-54.

44. Landon BE, Normand SLT, Blumenthal D, et al. Physician Clinical Performance Assessment: Prospects and Barriers. JAMA 2003;290:1183-9.

45. van Dijk L, Heerdink ER, Somai D, et al. Patient risk profiles and practice variation in nonadherence to antidepressants, antihypertensives and oral hypoglycemics. BMC Health Serv Res 2007;7:51.

46. Dharmarajan TS, Dharmarajan L. Tolerability of antihypertensive medications in older adults. Drugs Aging 2015;32:773-96.

47. Tamblyn R, Abrahamowicz M, Dauphinee D, et al. Influence of physicians' management and communication ability on patients' persistence with antihypertensive medication. Arch Intern Med 2010;170:1064-72.

48. Jacobs S, Ashcroft D, Hassell K. Culture in community pharmacy organisations: what can we glean from the literature? J Health Organ Manag 2011;25:420-54.

49. Scott T, Mannion R, Marshall M, et al. Does organisational culture influence health care performance? A review of the evidence. $J$ Health Serv Res Policy 2003;8:105-17.

50. Clark BE, Mount JK. Pharmacy service orientation: A measure of organizational culture in pharmacy practice sites. Res Soc Admin Pharm 2006;2:110-28. 